\title{
Relation between vit $D$ level and low antral follicle count in infertile female below 35 years
}

\author{
Seham A. El berry, A.N.El Mentawy, A.A.Sharaf EI Din, and H.M.Nagiub \\ Obstetrics and gynaecology, Dept., Faculty of Medicine, Benha University \\ E-mail:zafagodoctor@gmail.com
}

\begin{abstract}
Background: In the recent years, vitamin D has become a topical subject and a focus of research not only in reproductive medicine but across many medical disciplines. In reproductive medicine, studies have identified an association between vitamin D status in women and ovarian reserve, Aim and objectives: the aim of the study was to detect the relationship between vit D and low antral follicle count in infertile females below 35 years old, Subjects and methods: This was a cross-sectional observational study that was conducted at the outpatient clinic of obstetrics and gynecology in Benha university hospital and Tokh central hospital, The study was carried out on 50 infertile patients below 35 years old with low AFC diagnosed first by ultrasonography then vit d level was done for those patients to detect the relation between vit d and low AFC, Results: Patients' ages ranged from 22 to 34 years with mean of 25.98 years and median was 25 years. Mean \pm SD of AFC among participant women was $4.35 \pm 1.41$, ranged from 4 to 6 years and median was 4 . Mean \pm SD of Vitamin D was $18.67 \pm 14.58(\mathrm{ng} / \mathrm{ml})$ ranged from 13.5 to $28.2(\mathrm{ng} / \mathrm{ml})$ and median was 19.1 (ng/ml). There is a non-significant correlation between vitamin $\mathrm{D}$ and (Age, AFC and BMI).Conclusion: After conducting this study we could not found a significant correlation between vit D level and low antral follicle count. Future studies are required to investigate further interaction between vit D level and infertility.
\end{abstract}

Keywords: Vitamin D deficiency, ovarian reserve, Antral Follicle Count, Infertile.

\section{Introduction}

Vitamin D deficiency (VDD) is a major health problem in both the developed and developing countries across the world. Recent epidemiologic studies have shown relationships between low vitamin D levels have harmful effects on various systems and multiple disease states. Vitamin D is also responsible for expression of a large number of genes in reproductive tissues, implicating a role for vitamin $\mathrm{D}$ in female reproduction. Human and animal data suggest that low vitamin D status is associated with impaired fertility and endometriosis. Serum $25(\mathrm{OH}) \mathrm{D}$ provides the single best assessment of vitamin D status; it has a half-life of about 3 weeks, making it the most suitable indicator of vitamin D status. [1]

Vitamin D also inhibits cell proliferation and stimulates cell differentiation. This hormone primarily exerts its effects through the vitamin D receptor (VDR). Through its receptor, vitamin D can modify gene transcription, as well as protein and messenger ribonucleic acid (mRNA) production. In animal studies, dietary VDD leads to a $25 \%$ reduction in overall fertility. In various studies, the role of VDD has been correlated with polycystic ovary syndrome (PCOS) and a myriad of pregnancy-related disorders. [2]

Infertility is the failure of a couple to conceive after 1 year of regular, unprotected intercourse. Ovulatory disorder is one of the most common reasons of female factor infertility $30 \%$ of all cases). Reproductive aging is considered to be the consequence of a decrease in the quantity and quality of the ovarian follicle pool [3].

Autopsy studies of human ovaries show that the number of follicles decreases rapidly with female age, starting in fetal life and continuing until after menopause. However, between women of the same chronological age, the quantitative ovarian reserve may vary substantially. To assess the individual quantitative ovarian reserve, various ovarian reserve tests (ORTs) have been developed, viz. (1) day-3 follicle stimulating hormone (FSH), (2) anti-Mullerian hormone (AMH), and (3) antral follicle count (AFC). The number of antral follicles and the total ovarian volume as measured by transvaginal USG have been mentioned in the literature to predict declining fertility related to reproductive aging. [4]

This study aimed to detect the relationship between vit $\mathrm{D}$ and low antral follicle count in infertile females below 35 years old.

\section{Patients and methods}

This cross-sectional observational study had been conducted at the outpatient clinics of obstetrics and gynecology in Benha university hospital and Toukh central hospital. The protocol had been approved by the local ethics committee of obstetrics and gynecology department of Benha university hospital .verbal informed consent had been obtained from each patient before inclusion in the study.

The study had included 50 infertile patients below 35 years old with low AFC diagnosed first by transvaginal ultrasonography then vit $\mathrm{D}$ level had been done for those patients to detect the relation between vit $\mathrm{D}$ and low $\mathrm{AFC}$. 


\section{Inclusion criteria}

- All infertile females (1ry \&2ry) .

- below 35 years.

- low antral follicle count (less than 6 antral follicles in both ovary).

\section{Exclusion criteria}

- Patients above 35 years.

- Patients with normal antral follicle count.

- PCOS patients.

- Patients with any history of surgical procedures in ovaries.

- Fertile females.

All cases were subjected to the following

- History taking

- Clinical examination

- Abdominal and local clinical examination: was done to detect :

Scar of previous operation,

Mass, tenderness or rigidity,

Any abdominal or pelvic clinically detectable pathology.

- Investigations including: Measurement of vitamin D-level

Blood samples were taken to measure vitamin D concentrations within the first week following the initial consultation with a reproductive medicine specialist and at the same time of ultrasonographic assessment of the antral follicle count (AFC). Once the blood sample was taken for vitamin D concentration, the sample was centrifuged and frozen at $-20^{\circ}$ Celsius. All blood samples for vitamin D concentrations were measured by the ELISA assay at one time to avoid inter-assay variability, using electrochemiluminescence binding assay (Roche's test) and Cobas e immunoassay analyzers. Vitamin D deficiency was defined as serum level of $<20 \mathrm{ng} / \mathrm{ml} 25$ hydroxy vitamin D

- Imaging: Ultrasound for antral follicle count measurement

All patients were scanned by transvaginal $2 \mathrm{D}$ ultrasound on day $2-5$ of the cycle. Two ultrasound specialists were involved one from each hospital .The two involved investigators established a common ultrasound technique for the AFC measurement prior of commencing the study in order to avoid any bias through different techniques.

The scan was done by two sets voluson 730 pro V :GE Medical system with 7.5 MHZ endocavitary transducer 2D and LOGIQ F6 :GE Medical systems(CHINA) CO.LTD.

The optimal probe program, providing the best ultrasound image, was used in both fertility centers and the settings were maintained throughout the duration of the ultrasound evaluation. Each patient was asked to empty the bladder and was scanned in a Lloyd Davies position to ensure free manipulation of the transvaginal transducer.

Ultrasound assessment of the pelvis excluded pelvic pathology. The ovaries were visualized in the longitudinal plane and the number of antral follicles measuring 2-10 $\mathrm{mm}$ in diameter within each ovary was counted as the transducer was moved from one side of the ovary to the other. Two scans were obtained for each ovary and in case of any discrepancy between these two values, a third measurement was done to confirm the final value.

\section{Statistical Analysis}

Data collected throughout history, basic clinical examination, laboratory investigations and outcome measures coded, entered and analyzed using Microsoft Excel software. Data were then imported into Statistical Package for the Social Sciences (SPSS version 20.0) (Statistical Package for the Social Sciences) software for analysis. According to the type of data qualitative represent as number and percentage, quantitative continues group represent by mean $\pm \mathrm{SD}$, the following tests were used to test differences for significance;., correlation by Pearson's correlation or Spearman's . P value was set at $<0.05$ for significant results $\&<0.001$ for high significant result.

\section{Results}

Patients' ages ranged from 22 to 34 years with mean of 25.98 years and median was 25 years, BMI ranged from 23 to $34 \mathrm{~kg} / \mathrm{m}^{2}$ with mean of $28.28 \mathrm{~kg} / \mathrm{m}^{2}$ and median was $25 \mathrm{~kg} / \mathrm{m}^{2}$, table (1).

Mean \pm SD of Menarche age is $14.25 \pm 2.37$ years ranged from 10 to 14 years and median was 12 years, Mean \pm SD of Menstrual cycles are $31.2 \pm 5.22$ days ranged from 26 to 35 days and median was 28 days , and Mean \pm SD of Menstrual flow is $4.38 \pm 0.547$ days ranged from 2 to 6 days and median was 3 days table(2).

Table (1) Demographic data of the studied patients.

\begin{tabular}{lll}
\hline Variable & Patients & $(\mathbf{n}=\mathbf{5 0})$ \\
\hline Age $($ years $)$ & & \\
Mean \pm SD & $25.98 \pm 4.15$ \\
Median $($ range $)$ & $25(20-35)$ \\
BMI $\left(\mathbf{k g} / \mathbf{m}^{2}\right)$ & & \\
Mean \pm SD & $28.28 \pm 2.72$ \\
Median $($ range) & $30(23-34)$ \\
\hline
\end{tabular}


$2 \%$ of women were smokers, $4 \%$ had tubal factor, $10 \%$ had uterine fibroid removal, and $14 \%$ had IVF Table (3).

The majority of patients was 1ry type of infertility, and duration of infertility ranged from 3 to 7 years with mean of 3.69 years and median was 3 years, fig. (1).
Mean \pm SD of AFC among participant women was $4.35 \pm 1.41$, ranged from 4 to 6 years and median was 4 . Mean \pm SD of Vitamin $\mathrm{D}$ was $18.67 \pm$ $14.58(\mathrm{ng} / \mathrm{ml})$ ranged from 13.5 to $28.2(\mathrm{ng} / \mathrm{ml})$ and median was 19.1 (ng/ml), fig. (2).

There is a non-significant correlation between vitamin D and (Age, AFC and BMI) table (4).

Table (2) Gynecological data of the studied patients.

\begin{tabular}{lcc}
\hline Variable & Patients & $(\mathbf{n = 5 0})$ \\
\hline Menarche age (years) & $14.25 \pm 2.37$ \\
Mean \pm SD & $12(10-14)$ \\
Median (range) & & \\
Menstrual cycles (days) & $31.2 \pm 5.22$ \\
Mean \pm SD & $28(26-35)$ \\
Median (range) & & \\
Menstrual flow (days) & $4.38 \pm 0.547$ \\
Mean \pm SD & $3(2-6)$ \\
Median (range) & \\
\hline
\end{tabular}

Table (3) History distribution of the studied patients.

\begin{tabular}{lcc}
\hline Variable & Patients & (n=50) \\
\hline Smoker & $2(12 \%)$ & \\
Tubal factor & $2(4 \%)$ & \\
Uterine fibroid removal & $5(10 \%)$ & \\
IVF & $7(14 \%)$ \\
\hline
\end{tabular}

\section{Type}

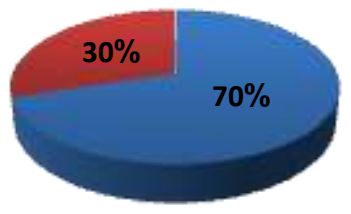

a 1 ry 2 ry

Fig. (1) Type of infertility.

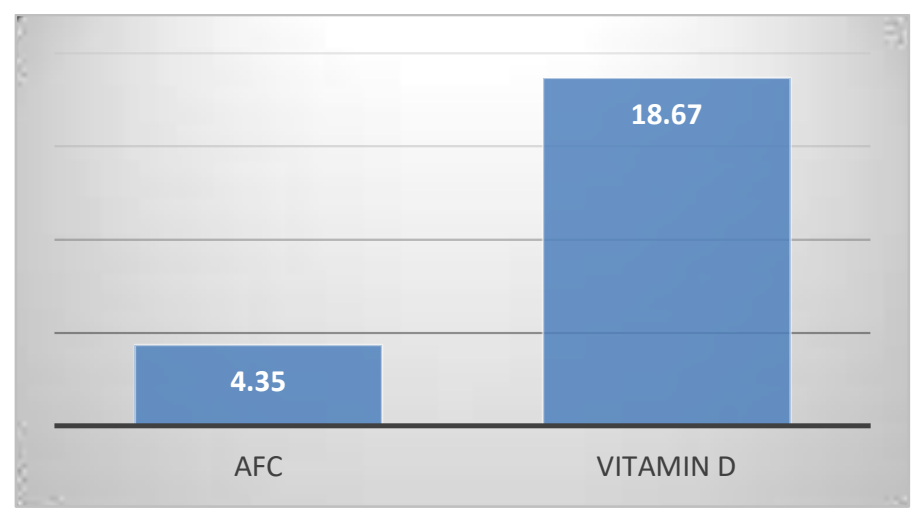

Fig. (2) AFC among participant women. 
Table (4) Correlation of vitamin D with other parameters of the studied patients.

\begin{tabular}{lcc}
\hline \multirow{2}{*}{ Variable } & \multicolumn{2}{c}{ Vitamin D } \\
& $\mathbf{r}$ & $\mathbf{P}$ \\
\hline Age & -0.103 & 0.496 \\
BMI & -0.126 & 0.236 \\
AFC & 0.232 & 0.311 \\
\hline
\end{tabular}

\section{Discussion}

This was a cross-sectional observational study that was conducted at the outpatient clinic of obstetrics and gynecology in Benha university hospital and Toukh central hospital, The study was carried out on 50 infertile patients below 35 years old with low AFC diagnosed first by ultrasonography then vit d level was done for those patients to detect the relation between vit $\mathrm{d}$ and low AFC.

The current study results are as follow : Mean \pm SD of AFC among participant women was $4.35 \pm 1.41$ (follicle), and Mean \pm SD of Vitamin D was $18.67 \pm$ 14.58 (ng/ml).

The current study results are in the line with those of other studies, who could not find a definite correlation between vit D level and AFC [5]-[7]. On the contrary, our results contrast with the literature reports that have found a negative correlation between vit D and AFC [8].

In the study of Drakopoulos et al., [5] who was investigating the relation between vit $\mathrm{D}$ level on ovarian reserve, the study included 283 consecutive infertile women younger than 42 years old and undergoing their first treatment cycle in their institution. All patients were recruited within a time interval of 12 months from the initiation of the study, before undergoing infertility treatment. Women consuming vitamin D supplements or taking medication for systematic disease or women who had undergone ovarian surgery were excluded from the study. All infertile women had serum AMH and vitamin D sampled on the same day. AFC was measured on the second or third day of the first cycle following the blood sampling for the determination of $\mathrm{AMH}$ and 25-OH vitamin D levels.

Main results and the role of chance: Among all patients, $30.7 \%(\mathrm{n}=87)$ were vitamin $\mathrm{D}$ deficient $(<20$ $\mathrm{ng} / \mathrm{mL})$ whereas $69.3 \%(\mathrm{n}=196)$ had normal vitamin D levels $(\geq 20 \mathrm{ng} / \mathrm{mL})$. The mean AMH and AFC levels did not differ significantly between the two groups: AMH $3.9 \mu / \mathrm{L}( \pm 3.8)$ versus $4.3 \mu / \mathrm{L}( \pm 4.8)$, $(\mathrm{P}$ value $=$ $0.5)$ and AFC $13.9( \pm 13.3)$ versus $12.7( \pm 11.4),(\mathrm{P}=$ $0.7)$,respectively. No correlation was observed between 25-O H vitamin D and AMH (spearman's $\mathrm{r}=0.02, \mathrm{P}$ value $=0.7$ ) or AFC (spearman's $\mathrm{r}=-0.02, \mathrm{P}$ value $=$ 0.7). In multiple linear regression analysis, after adjusting for potential confounders (age, BMI, smoking status, infertility cause and season of blood sampling), the regression slope in all participants for total $25 \mathrm{OH}-\mathrm{D}$ predictinglog ${ }_{10} \mathrm{AMH}$ was 0.006 [standard error $(\mathrm{SE})=0.07, \mathrm{P}$ value $=0.9$ ]. Similarly, no significant association was observed between AFC and vitamin D levels, even after controlling for relevant co-variants (regression co-efficient -0.09. SE 0.08,P value $=0.2$ ) .

The study of Nazanin Alavi et al., [6] aimed to investigate the impact of vitamin D on AMH serum concentrations and AFC. 287 infertile women were included in the analysis with a mean age of $29.95 \pm 4.73 \mathrm{yr}(18-45 \mathrm{yr})$ and a mean Body mass index of $25.11 \pm 4.41 \mathrm{~kg} / \mathrm{m} 2$. The median $\mathrm{AMH}$ and vitamin D levels were 3.20 and $22.82 \mathrm{ng} / \mathrm{ml}$, respectively. Considering the cut-off level of $20 \mathrm{ng} / \mathrm{ml}$, $58.7 \%$ were vitamin D deficient. Regression analysis showed no association between AMH and vitamin D levels ( $p=0.161)$, even after adjusting for baseline variables $(p=0.182)$. A total of 120 patients had an $\mathrm{AFC}<6$ and 164 patients had $\mathrm{AFC} \geq 6$, which was not statistically different between the groups with normal level or deficient vitamin $\mathrm{D}(\mathrm{p}=0.133)$.

The study of Fabris et al., [7] which was investigating the relation between vit $\mathrm{D}$ level and ovarian reserve markers in oocyte donors could not find an obvious correlation between vit $\mathrm{D}$ and $\mathrm{AFC}$ and $\mathrm{AMH}$. The value between the AMH levels and the bioavailable vitamin $\mathrm{D}$ levels was $\mathrm{r}=0.059$. The relationship between $\mathrm{AFC}$ and vitamin $\mathrm{D}$ in its bioavailable form yielded a value of $\mathrm{r}=0.081(\mathrm{P}=$ 0.011).

In comparison with the study of Arefi et al., [8] which aimed to evaluate the vitamin D status in an infertile population in Iran and to correlate vitamin D concentrations with parameters such as female age, skin type, degree of skin concealment and antral follicle count (AFC) as parameter of the ovarian reserve, and reported that total of 189 patients have been included in this prospective observational study, the mean age of the study group was 32.21 years, and the mean BMI of the patients was $26.70 \mathrm{~kg} / \mathrm{m}^{2}$ with a range of $17.9-35.5 \mathrm{~kg} / \mathrm{m}^{2}$. The mean vit $\mathrm{D}$ was for study group was $15.46 \mathrm{ng} / \mathrm{ml}$ and while the mean AFC in all patients was 10.84 follicle. There was negative correlation between vit D level and AFC in the study group .

The contradictory finding between the studies may be attributed to the different ethnicities of the 
study population, having different socio-cultural religious habits and different dress-codes. Drakopoulos et al., [5] evaluated women with Caucasian ethnicity, which have been treated in an infertility center due to primary / secondary infertility in Belgium .the study of Nazanin Alavi et al. [6] was studying three hundred and five infertile Iranian women referred to the IVF Unit who had different ethnicity, different sociocultural habits and different study population .The study of Fabris et al., [7] studied Spanish patients who had Caucasian ethnicity in contrast to those mentioned in our study the described study population presents completely different ethnicity and secondly majority of the study population is dressed in dress-codes, which leaves less than $10 \%$ of the skin uncovered, thereby limiting the skin exposure to the sun dramatically. The study of Arefi et al., [8] was studying a larger study population of Iranian women with different sociocultural habits and different dess-codes.

\section{Conclusion}

After conducting this study we could not found a significant correlation between vit $\mathrm{D}$ level and low antral follicle count. Future studies are required to investigate further interaction between vit $\mathrm{D}$ level and infertility.

\section{References}

[1] P. J. Malloy and D. Feldman, "Genetic disorders and defects in vitamin D action," Rheum. Dis. Clin., vol. 38, no. 1, pp. 93-106, 2012.
[2] M. Irani, H. Minkoff, D. B. Seifer, and Z. Merhi, "Vitamin D increases serum levels of the soluble receptor for advanced glycation end products in women with PCOS," J. Clin. Endocrinol. Metab., vol. 99, no. 5, pp. E886-E890, 2014.

[3] M. Aubuchon, R. O. Burney, D. J. Schust, and M. W. M. Yao, "Infertility and assisted Reproductive technology," Berek Novak's Gynecol. Fifteenth ed Lippincott Williams Wilkins, a Wolters KLuwer Bus., vol.3, no. 4, pp. 56- 63, 2012.

[4] L. F. Bancsi, F. J. M. Broekmans, M. J. C. Eijkemans, F. H. de Jong, J. D. F. Habbema, and E. R. te Velde, "Predictors of poor ovarian response in in vitro fertilization: a prospective study comparing basal markers of ovarian reserve," Fertil. Steril., vol. 77, no. 2, pp. 328 336, 2002.

[5] P. Drakopoulos et al., "The effect of serum vitamin D levels on ovarian reserve markers: a prospective cross-sectional study," Hum. Reprod., vol. 32, no. 1, pp. 208-214, 2017.

[6] N. Alavi, M. Ebrahimi, and F. Akbari-Asbagh, "The effect of vitamin D status on ovarian reserve markers in infertile women: A prospective crosssectional study," Int. J. Reprod. Biomed., vol. 18, no. 2, p. $85,2020$.

[7] A. M. Fabris et al., "Impact of vitamin D levels on ovarian reserve and ovarian response to ovarian stimulation in oocyte donors," Reprod. Biomed. Online, vol. 35, no. 2, pp. 139-144, 2017.

[8] S. Arefi et al., "Is the ovarian reserve influenced by vitamin $\mathrm{D}$ deficiency and the dress code in an infertile Iranian population?," J. Ovarian Res., vol. 11, no. 1, pp. 1-6, 2018. 